\title{
Pesquería y parámetros biométricos de los cangrejos del género Calappa (Brachyura: Calappidae) en el oriente de Venezuela
}

José J. Alió, Luis A. Marcano \& Douglas E. Altuve

Ministerio de Ciencia y Tecnología, Instituto Nacional de Investigaciones Agrícolas, Centro de Investigaciones Agrícolas de los Estados Sucre y Nueva Esparta, Aptdo. 236, Cumaná, edo. Sucre 6101 Venezuela. FAX 582934325385 ; jalio@inia.gov.ve

Recibido 10-I-2003. Corregido 15-VI-2005. Aceptado 07-VII-2005.

\begin{abstract}
Fishery and biometrics of genus Calappa crabs (Brachyura: Calappidae) in northeastern Venezuela. The box crabs, genus Calappa, are important crab species landed by the industrial trawl fleet in Venezuela. These crabs have a wide distribution in the country, from the Gulf of Venezuela to the Orinoco River, but major landings take place in such gulf, near Margarita Island and northern Sucre State. Average annual landing in the eastern region was $69 \mathrm{t}$ during 1970-2001, with a maximum of $221 \mathrm{t}$. For this study a sample of 2398 box crabs was collected by observers on board of industrial trawl vessels, which operated in eastern Venezuela from 1994 to 2000. Three species were found in the landings, Calappa sulcata and C. flammea, each represented $47 \%$ of the sample, and C. nítida, which accounted for $5 \%$ of the sample. Sex ratio was strongly biased towards males in C. sulcata (2.8:1) and in C. flammea (1.9:1), while it was only slightly biased in C. nitida (1.4:1). Due to its large size, C. sulcata is of great commercial interest, with average weight $290 \mathrm{~g}$ and a maximum weight of $720 \mathrm{~g}$; C. flammea showed an average weight of $140 \mathrm{~g}$ and a maximum weight of $418 \mathrm{~g}$, while C. nitida is the smallest species, with average weight $46 \mathrm{~g}$ and maximum weight not beyond $113 \mathrm{~g}$. Box crabs were captured by day and night in similar proportions, and were found in a wide depth range, from a few meters to $126 \mathrm{~m}$ deep; however, the largest captures were obtained in the interval 38 - $54 \mathrm{~m}$. Regressions between weight of chelae and total weight were linear, and significantly different between sexes. The relation between total weight and carapace width was allometric, with a power exponent $c a$. three in C. sulcata, and less than three in C. flamea and C. nítida. Females of $C$. sulcata and C. nitida showed power exponents significantly smaller than males. Since claws are removed from the animal to be commercialized, while the rest of the animal is returned alive to the sea, for practical purposes a common equation among species and sexes to convert claws weight into total body weight is: total weight $=3 \mathrm{x}$ claws weight. The relationship between catch per unit of effort (CPUE) and effort did not show a significantly decreasing trend, but landings follow the effort trend, with a decreasing tendency in recent years. No mature females were found in any of the three species, thus there could be reproductive migrations towards deeper waters from October to January, when abundance of crabs is lower in the study area. It is recommended that the practice of exploiting only one claw, returning the rest of the animal alive to the sea, should be promoted among fishers, while the removal of claws from female crabs should be avoided. Rev. Biol. Trop. 53(3-4): 463-474. Epub 2005 Oct 3.
\end{abstract}

Key words: Calappa, trawl fishery, northeastern Venezuela, biometry.

Los cangrejos del género Calappa Weber 1795, tienen una amplia distribución en los océanos del mundo, encontrándose en profundidades de 0 a $380 \mathrm{~m}$ (Galil 1997). En América se distribuyen desde Carolina del Norte y el Golfo de México, las Antillas hasta el Sur de Brasil (Rodríguez 1980, Rosas et al. 1992, Nakamura y De Loyola e Silva 1992).
Se estima que los actuales miembros de la familia Calappidae derivan de cangrejos que habitaban aguas profundas (Bellwood 1998). Su importancia ecológica radica en sus hábitos alimenticios, pues constituyen especies depredadoras de numerosos moluscos gasterópodos y bivalvos (Vermeij 1982, Signor 1985, Hughes y Elner 1989), así como de cangrejos 
ermitaños. Su interés también está asociado a que miembros de estas familias pueden ser hospederos del baculovirus de la mancha blanca (white spot syndrome virus ó WSSV), el cual ha causado importantes pérdidas económicas en instalaciones de cultivo de camarones (Flegel y Alday-Sanz 1998, Chakraborty et al. 2002, Hameed et al. 2003).

La carne de los cangrejos moros es muy apetecida, por lo que sus poblaciones son explotadas comercialmente con sistemas de arrastre (Aravindakshan y Sundaram 1983) ó con nasas (Ivo et al. 1996). Por su parte, Hayashi et al. (1993) destacaron que los descartes de cangrejos de los géneros Calappa y Ovalipes, tienen alto potencial como fuentes potenciales para la fabricación de aromatizantes para alimentos, más que otras especies de cangrejos.

Rodríguez y Suárez (2002) informan en Venezuela siete especies de cangrejo moro: $C$ sulcata Rathbun, 1898; C. cinerea Holthuis, 1958; C. ocellata Holthuis, 1958; C. flamea (Herbst, 1758); C. nítida Holthuis, 1958; C. angusta A. Milne Edwards, 1880 y C. tortugae. Por otra parte, Takeda y Okutami (1983), en un trabajo sobre muestreos de arrastre en las costas de Surinam y Guayana Francesa, informan a $C$. sulcata, C. flammea, C. nítida y C. angusta.

Los desembarques de cangrejos moros que se reportan en Venezuela son una mezcla de varias de estas especies. Las descargas promedio en el oriente del país se elevan a $69 \mathrm{t}$ por año, pero en la región occidental del país no se ha podido cuantificar su aporte, pues se desembarca una mezcla de cangrejos moros y portúnidos (R. Alvarez, Instituto Nacional de Investigaciones Agrícolas, com. pers.). Las tripulaciones suelen desprender las dos quelas de los cangrejos para su comercialización, siendo el animal vivo devuelto al mar. Recursos sometidos a este tipo de explotación, han sido denominados como reusables (Ehrhardt y Restrepo 1989). Sin embargo, hay que destacar que el desprendimiento de las quelas que se hace a bordo de las embarcaciones de arrastre, puede provocar desgarramiento y sangramiento persistente, si no ocurre por las suturas naturales para la autotomización; en la base (base-isquio) de los apéndices (Mantel 1983). El desprendimiento incorrecto de las quelas, puede causar la muerte por perdida excesiva de fluidos, o colateralmente regeneración tardía de estas como lo plantea (Kuris y Mager 1975), derivando en incapacidad para defenderse adecuadamente de otros depredadores o alimentarse. Por otra parte, aunque se desconoce la tasa de sobrevivencia luego que son devueltos al mar, se presume que los niveles de pesquerías podrían estar relacionados con ella. Es así como Davis et al. (1979 citado por Restrepo 1992) estimaron que en la pesquería del cangrejo Menippe mercenaria, de Florida, la tasa de sobrevivencia alcanza hasta un $50 \%$ como consecuencia del tiempo y condiciones en que los animales son mantenidos a bordo antes de ser devueltos al mar, así como con la magnitud del daño causado al cangrejo durante el desprendimiento de la quela. Debe destacarse que en esta pesquería no se permite la explotación del cangrejo completo, sino el desprendimiento de una o las dos quelas siempre y cuando tengan una longitud mayor de $7 \mathrm{~cm}$, permitiendo que el animal pueda defenderse y alimentarse con la que le queda (Muller y Bert 2001).

Con base en lo anterior, se consideró pertinente evaluar en el presente trabajo la composición de las pesquerías de cangrejos moros en el oriente de Venezuela y las tendencias en las pesquerías de estas especies, como complemento a los estudios sobre las especies capturadas incidentalmente por la flota arrastrera industrial camaronera. Se destacan además, las relaciones biométricas que permiten estimar las capturas generales de cangrejos moros, a partir de los desembarques de quelas.

\section{MATERIALES Y MÉTODOS}

Los muestreos fueron efectuados por observadores científicos a bordo de embarcaciones industriales de arrastre entre 1994 y 2000. Las campañas se efectuaron en las zonas de pesca comprendidas en la región nororiental de Venezuela: plataforma Unare

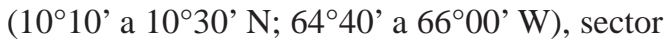


norte de la isla de Margarita $\left(11^{\circ} 00^{\prime}\right.$ a $11^{\circ} 20^{\prime}$ $\mathrm{N}$; 6330' a 64³0' W), sector norte de la Península de Paria (1050' a $11^{\circ} 30^{\prime} \mathrm{N}$; 61 6 $50^{\circ}$ a 6330' W), Golfo de Paria (1000' a $10^{\circ} 30^{\prime}$ $\mathrm{N}$; $61^{\circ} 55^{\prime}$ a $62^{\circ} 25^{\prime} \mathrm{W}$ ) y frente al delta del río Orinoco ( $8^{\circ} 30^{\prime}$ a $10^{\circ} 00^{\prime} \mathrm{N}$; 59 $20^{\circ}$ a $62^{\circ} 00^{\prime}$ W). Los observadores recogían los cangrejos de talla comercial y completaban empaques de $10 \mathrm{~kg}$, los cuales eran congelados o preservados en hielo a bordo, y trasladados para su procesamiento en el laboratorio del Instituto Nacional de Investigaciones Agrícolas (INIA) en Cumaná.

En el laboratorio se identificó la especie de cada ejemplar, de acuerdo a la clave de Rodríguez (1980) y a las figuras de Takeda y Okutami (1983). Así mismo, se identificó el sexo de cada ejemplar de acuerdo al ancho del abdomen, y se registraba la presencia de hembras ovadas. A cada ejemplar se le se estimó el peso total, peso del cuerpo y de las quelas, en una balanza electrónica Ohaus de $0.5 \mathrm{~g}$ de precisión. Adicionalmente, se midieron la longitud anteroposterior y el ancho del caparazón, con un vernier de $0.1 \mathrm{~mm}$ de precisión.

La información sobre las descargas de cangrejos moros por parte de las embarcaciones industriales de arrastre entre 1970 y 2001, provino de los registros recabados por Instituto Nacional de Pesca y Acuicultura (INAPESCA) en Cumaná, en base a las planillas de descarga y bitácoras de pesca que entregan las embarcaciones en el puerto de desembarque. Adicionalmente, se contó con la información sobre las capturas registrada por los observadores a bordo. En el INIA, en colaboración con el INAPESCA, se realizó la trascripción y el procesamiento de la información pesquera. Los desembarques se expresaron como biomasa total de cangrejos; cuando se desembarcaron quelas, el peso fue multiplicado por 3 para convertirlo a peso total de cangrejos, de acuerdo a lo encontrado en evaluaciones preliminares realizadas en el INIA.

Las bases de datos fueron creadas en la hoja de cálculo MS-Excel 2000 y los análisis estadísticos fueron efectuados en esa misma hoja de cálculo. Para el análisis de la estacionalidad de las capturas se dispuso de información para los períodos 1976-79, 1981-82, 1987-88, 1991-97 y 2001, mientras que para el análisis de la estacionalidad del esfuerzo se evaluaron los períodos 1976-83, 1987-97 y 2001. En este sentido, los datos mensuales fueron estandarizados, sustrayéndose del valor de cada mes el promedio del respectivo año y el resultado se dividió entre la desviación estándar de dicho año. Una vez estandarizados los datos, se estimó el promedio y el error estándar de cada mes en la serie de años considerada. El análisis de covarianza para comparar pendientes e interceptos de múltiples regresiones lineales fue programado en Fortran IV, de acuerdo al procedimiento de cálculo sugerido por Zar (1996).

\section{RESULTADOS}

Los desembarques de cangrejos moros en la región oriental del país variaron entre 7 y 221 t durante el período 1970-2001 (Fig. 1). Sus niveles se mantuvieron por debajo de $20 \mathrm{t}$ por año hasta 1979, duplicándose en 1980-86 y alcanzando un máximo entre 1987 y 89. Desde entonces se observa una declinación sostenida de los desembarques, interrumpida por elevadas capturas en 1995. Se observó una marcada estacionalidad de los desembarques, con los valores más elevados durante marzo-abril y junio-octubre, y los menores entre octubre y enero, siguiendo variaciones similares en el esfuerzo de pesca (Fig. 2). La CPUE en la región oriental del país mostró una relación ascendente significativa con el esfuerzo de pesca (Fig. 3).

Se procesó una muestra de 2398 ejemplares del género Calappa. En la misma se observaron tres especies, dos en proporción similar de $47 \%$, C. sulcata y C. flamea, y una tercera, C. nítida, con una representación de 5\% (Cuadro 1). La proporción de sexos estuvo significativamente sesgada hacia los machos en las tres especies (Cuadro 1). No se encontraron hembras ovadas.

Las capturas de cangrejos moros no mostraron diferencias significativas entre el día 


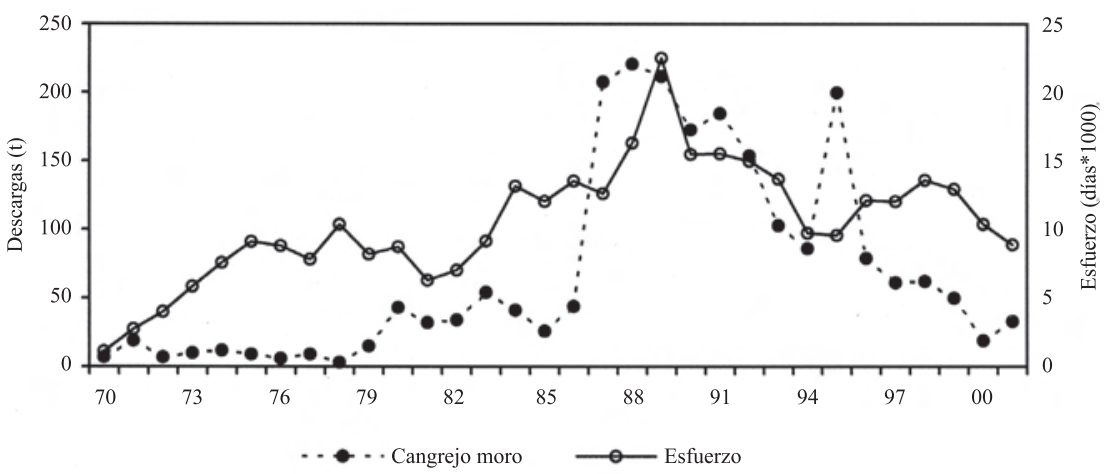

Fig. 1. Desembarques de cangrejo moro, Calappa spp. y esfuerzo desarrollado por la flota arrastrera industrial que opera en la región oriental de Venezuela durante 1970-2001 (Fuente: L. Marcano, INIA Ctro. Invest. Agric. Sucre - Nueva Esparta).

Fig. 1. Landings of box crab, Calappa spp., and effort made by the industrial trawl fleet operating in eastern Venezuela during 1970-2001 (Source: L. Marcano, INIA Ctro. Invest. Agric. Sucre - Nueva Esparta).

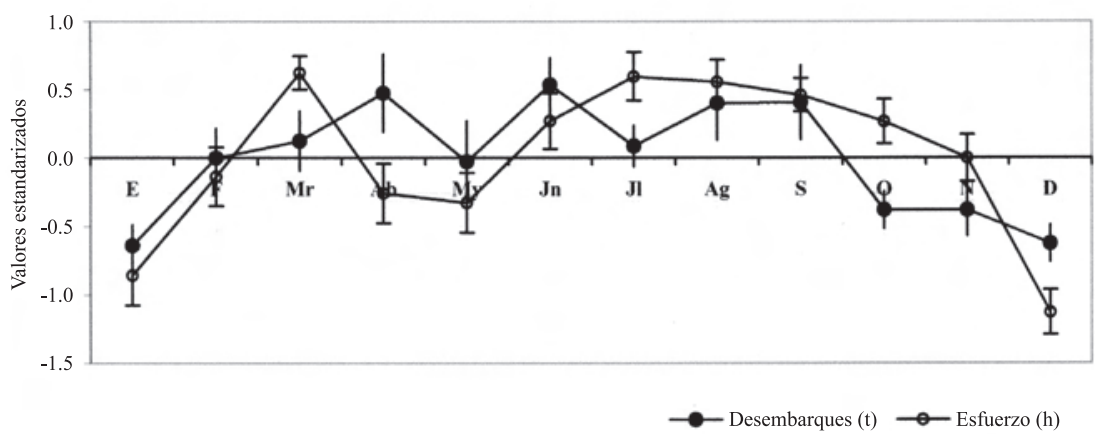

Fig. 2. Variación estacional (promedio \pm EE) de las descargas de cangrejo moro y del esfuerzo de pesca de la flota de arrastre industrial que opera en la región oriental de Venezuela, durante el período 1976-2001 (Fuente: L. Marcano, INIA Cetr. Invest. Agric. Sucre - Nueva Esparta).

Fig. 2. Seasonal variation (mean $\pm \mathrm{EE}$ ) of box crab landings and fishing effort of the industrial trawl fleet operating in eastern Venezuela during 1976-2001 (Source: L. Marcano, INIA Cetr. Invest. Agric. Sucre - Nueva Esparta).

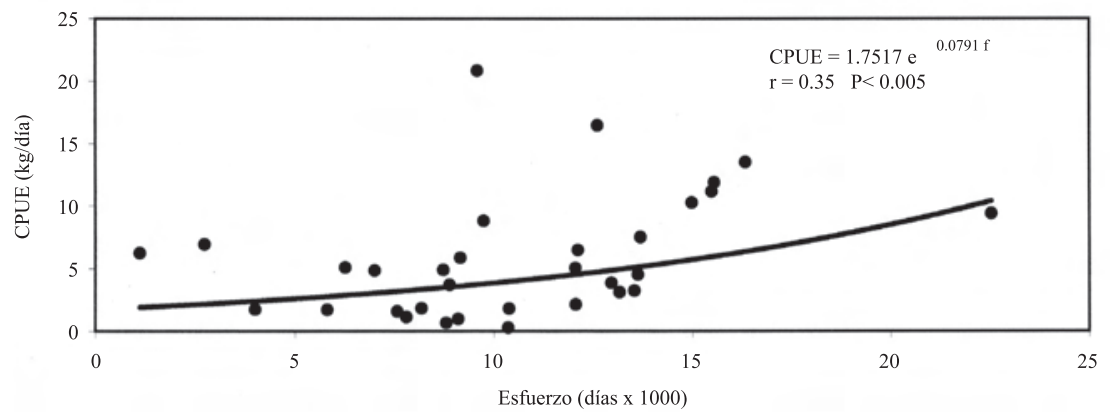

Fig. 3. Variación de la CPUE de cangrejo moro con el esfuerzo de pesca de la flota de arrastre industrial que opera en la región oriental del país, durante el período 1970-2001. (Fuente: L. Marcano, INIA Ctr. Invest. Agric. Sucre - Nueva Esparta).

Fig. 3. Variation of CPUE of box crab with the fishing effort of the industrial trawl flete operating in eastern Venezuela during 1970-2001 (Source: L. Marcano, INIA Cetr. Invest. Agric. Sucre - Nueva Esparta). 
CUADRO 1

Proporción de especies y relación de sexos en los desembarques de cangrejo moro evaluados por observadores, a bordo de embarcaciones industriales de arrastre que operaron en la región oriental de Venezuela entre 1994 y 2000

TABLE 1

Proportion of species and sex ratio in the landings of box crab evaluated by observers, on board of industrial trawl vessels operating in eastern Venezuela between 1994 and 2000

\begin{tabular}{lcccccc}
\multicolumn{1}{r}{ Especie } & Machos & Hembras & Total & $\%$ & M:H & $X^{2}$ \\
C. sulcata & 829 & 295 & 1124 & 46.9 & 2.8 & $\mathrm{P}<0.001$ \\
C. flammea & 739 & 399 & 1138 & 47.5 & 1.9 & $\mathrm{P}<0.001$ \\
C. nitida & 80 & 56 & 136 & 5.7 & 1.4 & $\mathrm{P}<0.05$ \\
Total & & & 2398 & 100 & &
\end{tabular}

(desembarques de $180 \mathrm{~kg}$ para un esfuerzo de 1086 horas) y la noche (desembarques de $290 \mathrm{~kg}$ para un esfuerzo de 1899 h; $X^{2}=0,64 ; \mathrm{P}>0.05$ ). Las mayores capturas de las tres especies tuvieron lugar a profundidades de 38 a 54 m (20 a 30 brazas; Cuadro 2). Calappa sulcata tuvo una distribución más amplia, entre pocos metros y $126 \mathrm{~m}$ de profundidad, mientras que $C$. flammea y $C$. nitida se observaron solamente entre 20 y 72 m (11 a 40 brazas). La mayor proporción del esfuerzo se desarrolló entre 20 y 36 m (11 a 20 brazas), y la heterogeneidad entre la distribución batimétrica del esfuerzo y las capturas fue altamente significativa $\left(\chi^{2}, \mathrm{P}<0.001\right)$.

La distribución geográfica de los cangrejos moros indica que se encuentran presentes en todo el oriente del país, desde la plataforma Unare-Píritu hasta la región cercana al Río
Esequibo (Fig. 4, 5 y 6). La mayoría de los ejemplares de las tres especies fueron capturados en los alrededores de la Isla de Margarita y Norte de la península de Paria. No se reportaron capturas en el Golfo de Paria. Calappa sulcata mostró una distribución más amplia, encontrándose tanto el Mar Caribe oriental como en la zona Atlántica de Venezuela (Fig. 4). Por su parte, C. flammea (Fig. 5) and C. nitida (Fig. 6) mostraron una distribución similar en el Mar Caribe oriental, no siendo capturadas en la zona Atlántica del país.

Las relaciones biométricas entre el peso total de los ejemplares en función del peso de las quelas para C. sulcata, C. flammea y $C$. nítida fueron lineales (Cuadro 3). Las diferencias de pendientes de las regresiones entre sexos fueron muy significativas en $C$. sulcata

CUADRO 2

Distribución por intervalo de profundidad del esfuerzo de pesca y las capturas (número) de cangrejos moros en el oriente de Venezuela durante 1994-2000

TABLE 2

Distribution by depth range of the fishing effort and catch (number) of box crabs in eastern Venezuela during 1994-2000

\begin{tabular}{ccccccccc} 
Int. Prof. & \multicolumn{2}{c}{ Esfuerzo } & \multicolumn{2}{c}{ C. sulcata } & \multicolumn{2}{c}{ C. flammea } & \multicolumn{2}{c}{ C. nitida } \\
$(\mathrm{m})$ & $(\mathrm{h})$ & $\%$ & $\mathrm{~N}$ & $\%$ & $\mathrm{~N}$ & $\%$ & $\mathrm{~N}$ & $\%$ \\
$0-19$ & 1524 & 7.9 & 41 & 4 & & & & \\
$20-37$ & 10506 & 54.6 & 387 & 34 & 455 & 40 & 25 & 22 \\
$38-55$ & 5289 & 27.5 & 543 & 48 & 655 & 58 & 86 & 76 \\
$56-73$ & 1032 & 5.4 & 61 & 5 & 28 & 2 & 2 & 2 \\
$74-91$ & 702 & 3.7 & 85 & 8 & & & & \\
$92-109$ & 12 & 0.1 & & & & & & \\
$110-126$ & 162 & 0.8 & 15 & 1 & & & & \\
Total & 19227 & 100.0 & 1132 & 100 & 1138 & 100 & 113 & 100
\end{tabular}




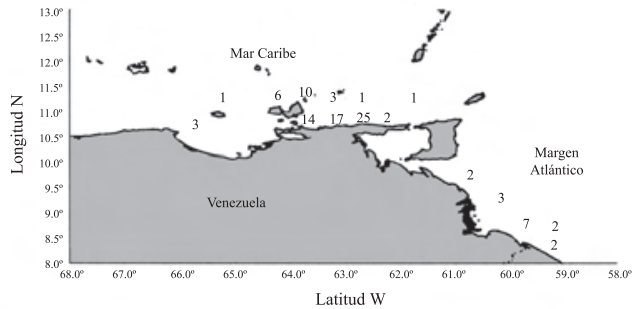

Fig. 4. Distribución porcentual de las capturas de $C$. sulcata en el nororiente de Venezuela, por cuadrantes de pesca de 55.6 × $55.6 \mathrm{~km}$ ( 30 x 30 millas $)$ durante el período 1994-2000.

Fig. 4. Percent distribution of catches of C. sulcata, in northeastern Venezuela, by fishing squares of $55.6 \mathrm{x}$ $55.6 \mathrm{~km}(30 \times 30$ miles) during the period 1994-2000.

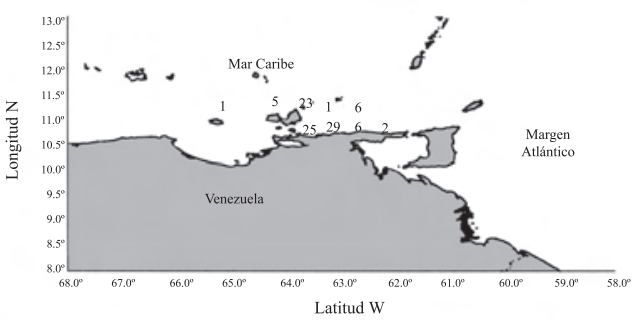

Fig. 5. Distribución porcentual de las capturas de C. flammea en el nororiente de Venezuela, por cuadrantes de pesca de $55.6 \times 55.6 \mathrm{~km}(30 \times 30$ millas $)$ durante el período 1994-2000.

Fig. 5. Percent distribution of catches of C. flammea, in northeastern Venezuela, by fishing squares of $55.6 \mathrm{x}$ $55.6 \mathrm{~km}$ ( $30 \times 30$ miles) during the period 1994-2000.

La estructura de tamaños difirió apreciablemente entre especies y entre sexos (Cuadro 4). Los ejemplares de C. sulcata fueron apreciablemente más corpulentos que los de las otras dos especies, pudiendo llegar a alcanzar pesos totales de casi $3 / 4 \mathrm{~kg}(720 \mathrm{~g})$. Los ejemplares de C. flammea presentaron pesos de tamaño intermedio, llegando a alcanzar $418 \mathrm{~g}$. Por su parte, los ejemplares de C. nítida fueron relativamente pequeños, con pesos totales que no superaron los $113 \mathrm{~g}$. Las hembras de estas tres especies fueron menores que los machos (Figs. 7-9). Fue poco frecuente encontrar cangrejos pequeños como componentes de la fauna descartada, siendo comercializados la mayoría de los cangrejos moros capturados. Los ejemplares de C. sulcata menores de $100 \mathrm{~g}$ y C. flammea (Prueba t-Student, $\mathrm{P}<0.001$ ). La comparación de pendientes entre sexos de $C$. nítida no indicó una diferencia significativa (ANCOVA, $\mathrm{P}>0.05$ ), pero la diferencia resultó ser muy significativa al comparar los interceptos (ANCOVA, $\mathrm{P}<0.001$ ). Una aproximación para estimar el peso total de los ejemplares capturados de cualquiera de las tres especies, en función del peso de las quelas, resultó de aplicar la siguiente ecuación:
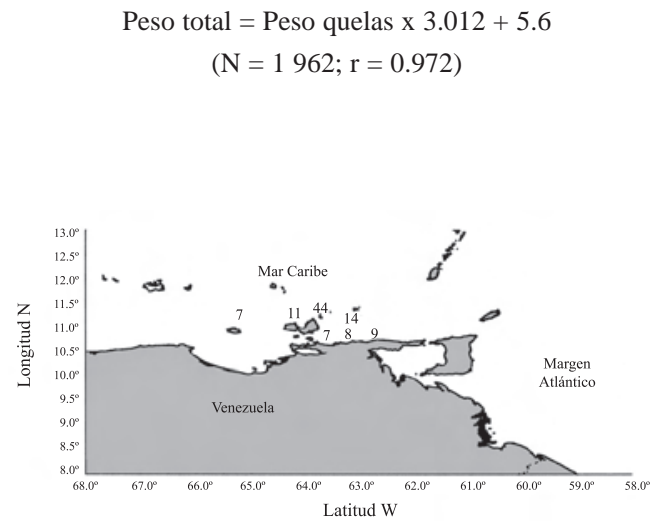

Fig. 6. Distribución porcentual de las capturas de $C$. nitida en el nororiente de Venezuela, por cuadrantes de pesca de 55.6 × $55.6 \mathrm{~km}(30 \times 30$ millas $)$ durante el período 1994-2000.

Fig. 6. Percent distribution of catches of C. nitida, in northeastern Venezuela, by fishing squares of 55.6 x $55.6 \mathrm{~km} \mathrm{(30}$ x 30 miles) during the period 1994-2000.

representaron menos de $10 \%$ en las capturas, mientras que los de C. flammea representaron un $26 \%$ en las capturas.

La relación entre el peso total y el ancho del caparazón se describió mediante una ecuación alométrica, del tipo Peso total $=\mathrm{a} *$ Ancho del caparazón ^ b, con un exponente " $b$ " cercano a tres en el caso de $C$. sulcata, y alométrica minorante, con un exponente " $\mathrm{b}$ " menor que tres, en los casos de C. flamea y C. nítida (Cuadro 5). Las diferencias de pendientes entre sexos fueron significativas para C. sulcata y C. nítida (Prueba t-Student, $\mathrm{P}<0,05$ y $\mathrm{P}<0,01$, respectivamente), mostraron las hembras exponentes significativamente menores a los machos. No se encontró diferencia significativa entre sexos en C. flammea. 
CUADRO 3

Relaciones biométricas entre el peso total ( $g$ ) y el peso de las quelas de C. sulcata, C. flammea y C. nitida

TABLE 3

Biometric relations between total weight ( $g$ ) and weight of chelae of C. sulcata, C. flammea and C. nitida

Peso quelas $=\mathrm{a}$ Peso Total $+\mathrm{b}(1)$

\begin{tabular}{|c|c|c|c|c|c|}
\hline & Sexo & $\mathrm{a}$ & b & $\mathrm{r}$ & $\mathrm{N}$ \\
\hline \multirow{3}{*}{ C. sulcata } & M & 3.665 & 0.306 & 0.910 & 662 \\
\hline & $\mathrm{H}$ & -3.575 & 0.357 & 0.904 & 370 \\
\hline & Común & 0.938 & 0.322 & 0.911 & 1032 \\
\hline \multirow{3}{*}{ C. flammea } & M & 3.665 & 0.306 & 0.910 & 662 \\
\hline & $\mathrm{H}$ & -3.575 & 0.357 & 0.904 & 370 \\
\hline & Común & 0.938 & 0.322 & 0.911 & 1032 \\
\hline \multirow{3}{*}{ C. nitida } & M & 1.613 & 0.308 & 0,968 & 55 \\
\hline & $\mathrm{H}$ & 1.271 & 0.274 & 0,907 & 50 \\
\hline & Común & 1.476 & 0.315 & 0.959 & 105 \\
\hline & \multicolumn{5}{|c|}{ Peso total $=\mathrm{c}$ Peso quelas $+\mathrm{d}(2)$} \\
\hline & & $\mathrm{c}$ & d & $\mathrm{r}$ & $\mathrm{N}$ \\
\hline \multirow{3}{*}{ C. sulcata } & M & 7.060 & 3.023 & 0.964 & 574 \\
\hline & $\mathrm{H}$ & 21.819 & 2.977 & 0.947 & 251 \\
\hline & Común & 15.324 & 2.972 & 0.964 & 825 \\
\hline \multirow{3}{*}{ C. flammea } & M & 17.687 & 2.681 & 0.910 & 662 \\
\hline & $\mathrm{H}$ & 29.610 & 2.285 & 0.904 & 370 \\
\hline & Común & 21.337 & 2.577 & 0.911 & 1032 \\
\hline \multirow{3}{*}{ C. nitida } & M & -1.347 & 3.043 & 0,968 & 55 \\
\hline & $\mathrm{H}$ & 3.084 & 3.005 & 0,907 & 50 \\
\hline & Común & 2.548 & 2.916 & 0.959 & 105 \\
\hline
\end{tabular}

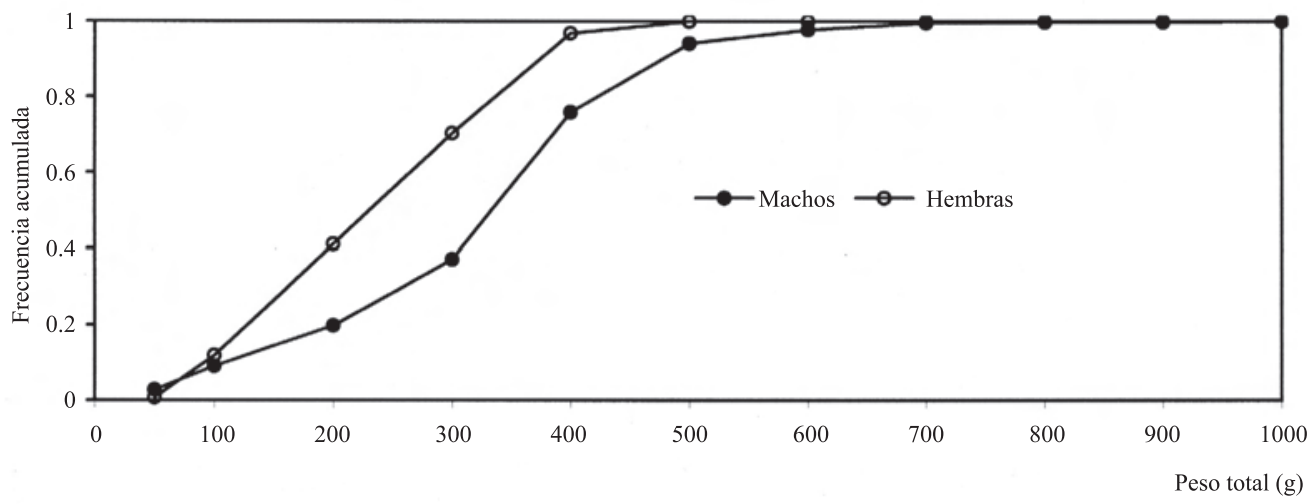

Fig. 7. Comparación de la estructura de tallas de machos y hembras de C. sulcata capturados por la flota de arrastre industrial en la región oriental de Venezuela.

Fig. 7. Comparison of the size structure of males and females of C. sulcata captured by the industrial fleet of trawlers in eastern Venezuela. 


\section{CUADRO 4}

Promedios de pesos totales $(\mathrm{g}$ ) de las especies de cangrejo moro capturados por las embarcaciones de arrastre industrial en la región oriental de Venezuela, durante 1994-2000

TABLE 4

Average weight ( $g$ ) of species of box crab captured by industrial trawl vessels in eastern Venezuela during 1994-2000

$\begin{array}{lcccc}\text { Especie } & \text { Sexo } & \text { Promedio } \pm \mathrm{DE} & \mathrm{N} & \text { Intervalo } \\ & \mathrm{M} & 317.7 \pm 133 & 580 & 29-712 \\ \text { C. } \text { sulcata } & \mathrm{H} & 233.5 \pm 102 & 253 & 41-491 \\ & \text { Común } & 292.1 \pm 130 & 833 & 29-712 \\ & & & & \\ \text { C. flammea } & \mathrm{M} & 153.4 \pm 135 & 664 & 32-418 \\ & \mathrm{H} & 116.6 \pm 49 & 371 & 27-308 \\ & \text { Común } & 140.2 \pm 58 & 1035 & 27-418 \\ & & & & \\ \text { C. nitida } & \mathrm{M} & 51.6 \pm 15 & 55 & 14-113 \\ & \mathrm{H} & 38.9 \pm 15 & 50 & 21-98 \\ & \text { Común } & 48.5 \pm 24 & 105 & 14-113\end{array}$

CUADRO 5

Relaciones biométricas entre el peso total (g) y el ancho del caparazón ( $\mathrm{mm}$ ) de las especies de cangrejo moro capturadas por la flota industrial de arrastre que opera en el oriente de Venezuela durante 1994-2000

TABLE 5

Biometric relations between total weight $(\mathrm{g})$ and carapace width $(\mathrm{mm})$ of the species of box crab captured by the industrial fleet of trawlers operating in eastern Venezuela during 1994-2000

\begin{tabular}{|c|c|c|c|c|c|c|}
\hline \multirow[b]{2}{*}{ Especie } & \multicolumn{5}{|c|}{ Peso total $=$ a Ancho caparazón ${ }^{\wedge} b$} & \multirow[b]{2}{*}{$\mathrm{p}$} \\
\hline & Sexo & a & $\mathrm{b}$ & $\mathrm{r}$ & $\mathrm{N}$ & \\
\hline \multirow{3}{*}{ C. sulcata } & M & $1.62610^{\wedge-4}$ & 3.069 & 0.934 & 552 & \multirow{3}{*}{$<0.05$} \\
\hline & $\mathrm{H}$ & $4.52110^{\wedge-4}$ & 2.849 & 0.896 & 247 & \\
\hline & Común & $2.21110^{\wedge-4}$ & 3.002 & 0.929 & 799 & \\
\hline \multirow{3}{*}{ C. flammea } & M & $5.23110^{\wedge-3}$ & 2.273 & 0.852 & 633 & \multirow{3}{*}{$<0.01$} \\
\hline & $\mathrm{H}$ & $3.35010^{\wedge-3}$ & 2.374 & 0.870 & 354 & \\
\hline & Común & $4.40810^{\wedge-3}$ & 2.311 & 0.878 & 986 & \\
\hline \multirow{3}{*}{ C. nitida } & M & $4.36410^{\wedge-4}$ & 2.798 & 0.904 & 51 & \multirow{3}{*}{$>0.05$} \\
\hline & $\mathrm{H}$ & $5.06010^{\wedge-2}$ & 1.611 & 0.566 & 48 & \\
\hline & Común & $1.08610^{\wedge-3}$ & 2.566 & 0.824 & 99 & \\
\hline
\end{tabular}

\section{DISCUSIÓN}

Las capturas de cangrejos moros en la región oriental del país estuvieron compuestas de tres especies, C. sulcata, C. flammea y $C$. nítida. Las dos primeras se encontraron en proporciones similares (ca. $47 \%$ ), mientras que la representación de C. nítida fue muy reducida $(5 \%)$. Al igual que el resto de las capturas incidentales asociadas a las pesquerías industriales de camarones, la de los cangrejos moros resulta de una explotación no dirigida hacia estos rubros. Las descargas se ven así afectadas por fallas en su reporte. Por otra parte, el esfuerzo 


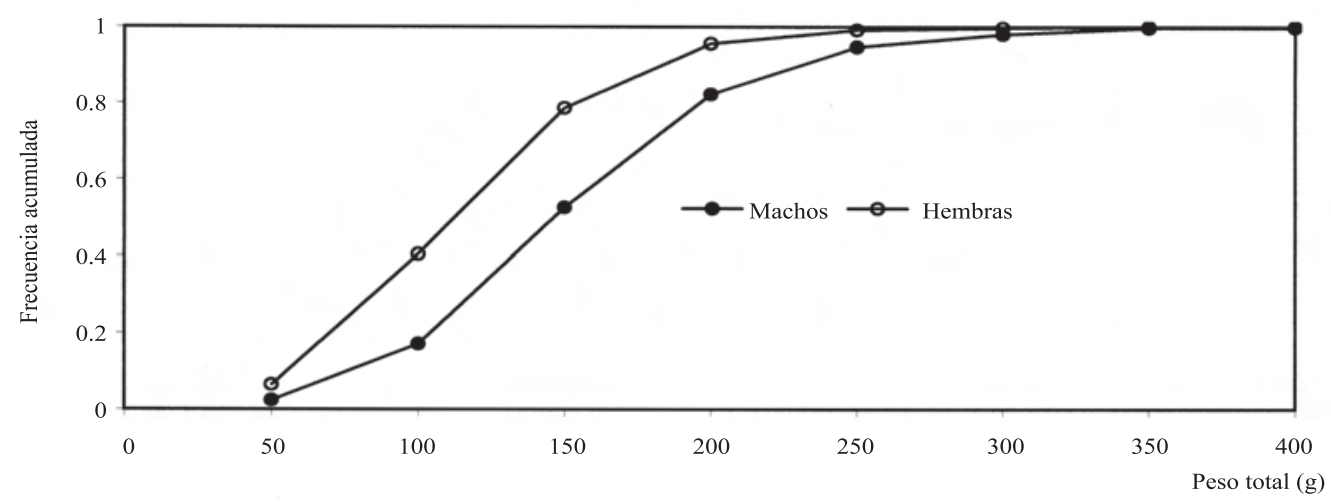

Fig. 8. Comparación de la estructura de tallas de machos y hembras de C. flammea capturados por la flota de arrastre industrial en la región oriental de Venezuela.

Fig. 8. Comparison of the size structure of males and females of C. flammea captured by the industrial fleet of trawlers in eastern Venezuela.

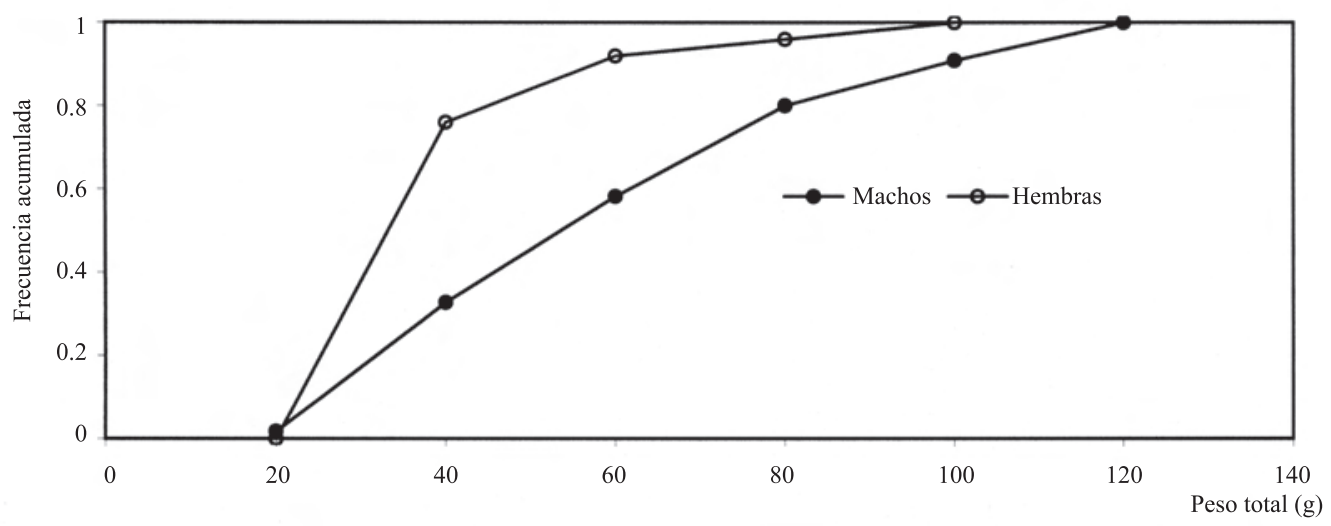

Fig. 9. Comparación de la estructura de tallas de machos y hembras de C. nitida capturados por la flota de arrastre industrial en la región oriental de Venezuela.

Fig. 9. Comparison of the size structure of males and females of C. nitida captured by the industrial fleet of trawlers in eastern Venezuela.

que realmente se hace sobre estos recursos debe ser menor al total que ejerce la flota, pero su magnitud se desconoce. Una posibilidad para estimarlo sería registrando la fracción del esfuerzo ejercido en las áreas de mayor abundancia de cangrejos moros.

El no encontrar hembras ovadas en las capturas indicaría que estas especies no se reproducen en la zona de operación de la flota arrastrera venezolana, restringida a los primeros $130 \mathrm{~m}$ de profundidad en la plataforma continental. Según Bellwood (1998) el origen de estos cangrejos estaría asociado a especies de aguas profundas, y su distribución es amplia en los primeros $400 \mathrm{~m}$ de profundidad. Por ello pudiera plantearse la posibilidad de que las tres especies de Calappa aquí estudiadas realicen migraciones reproductivas hacia aguas 
más profundas en el talud continental. El sesgo hacia los machos observado en la proporción de sexos en las tres especies indicaría que una mayor proporción de hembras pudiera encontrarse en aguas más profundas.

Las capturas de cangrejos se ven afectadas no solo por las variaciones en la abundancia de estos cangrejos, sino también por las del esfuerzo. En la región oriental de Venezuela existen dos períodos de veda al año de un mes de duración cada uno, entre el 15 diciembre al 15 de enero, y desde el 15 de mayo al 15 de junio. Se observan importantes descensos en las capturas durante los períodos en los que la flota ejerce menor esfuerzo, como diciembreenero y abril-mayo (Fig. 2). Sin embargo, el descenso observado en octubre y noviembre no parece corresponderse con marcados cambios en el esfuerzo de pesca, y más bien sugiere una disminución importante en la densidad de cangrejos en la región. Este patrón sugeriría que de existir una migración reproductiva de estos organismos hacia aguas más profundas, la misma estaría ocurriendo entre los meses de octubre y enero de cada año.

Debido a la relación positiva entre la CPUE y el esfuerzo de pesca de arrastre, observada en la región oriental, no se pudo estimar el rendimiento máximo sostenido de estas pesquerías ni el esfuerzo óptimo por medio de la utilización de modelos de rendimiento excedentario. Esta situación pudiera estar asociada, por una parte, a la deficiente estimación del esfuerzo de pesca que se ejerce realmente sobre los recursos de cangrejos moros; por la otra, a la manipulación peculiar que la flota de arrastreros hace de estos recursos. Al devolver a la mayoría de los ejemplares vivos al mar, la mortalidad por pesca resultaría ser menor que la de una pesquería tradicional en la cual todos los individuos extraídos mueren. Este hecho apoya la hipótesis de que la sobrevivencia de los cangrejos sin quelas puede ser elevada. Sin embargo, una confirmación del mismo pudiera efectuarse registrando la presencia de cangrejos vivos y sin quelas o con quelas en regeneración, que se encuentren en las capturas que efectúan los barcos arrastreros. Adicionalmente, debería evaluarse la sobrevivencia de cangrejos moros sin quelas ó con una quela, bajo condiciones controladas, como pudieran ser las de un corral en la zona costera. Por los momentos, la tradición de devolver los cangrejos vivos al mar, luego de remover las quelas, debe promoverse entre las tripulaciones de los barcos de arrastre. Adicionalmente, debería regularse que tan solo una de las quelas sea removida para asegurar una mayor sobrevivencia de los cangrejos una vez que son devueltos al mar. En general, debería evitarse la remoción de quelas de las hembras.

Los cangrejos moros presentan una amplia distribución en el oriente del país, desde zonas típicamente oceánicas, como los alrededores de la isla de Margarita, hasta aguas menos salinas como las presentes frente al delta del Río Orinoco. Las mayores capturas, que se registraron en las inmediaciones de la Isla de Margarita y del norte de Sucre, pueden estar asociadas a la abundancia particularmente elevada de moluscos bivalvos en dicha región (Mendoza et al. 1994), pues estos organismos constituyen parte importante de la dieta de los cangrejos moros (Hughes y Elner 1989).

De la muestra de cangrejos moros evaluada se desprende que los machos de C. sulcata y C. flamea tienen mayor interés comercial para la pesquería, debido a la talla más elevada que pueden alcanzar. Como norma de manejo, pudiera establecerse la liberación de las hembras sin remoción de sus quelas, tal como se observa en otras pesquerías de cangrejos en Venezuela, como la del Callinectes spp. en el Lago de Maracaibo, en la que todas las hembras capturadas son liberadas vivas (Villasmil y Mendoza 2001). El hecho que la distribución de sexos se encontró fuertemente sesgada hacia los machos en la especie de mayor interés comercial, C. sulcata, facilitaría esta labor.

Las descargas de cangrejos moros están constituidas por quelas y en menor proporción por ejemplares completos. Surge así la necesidad de convertir la biomasa de quelas a peso total de los ejemplares. Las relaciones biométricas entre el peso de las quelas y el peso total de los ejemplares muestran diferencias 
importantes entre las tres especies evaluadas y entre sexos. Sin embargo, las descargas de quelas provienen de ejemplares de las tres especies mezcladas, resultando difícil la identidad de las mismas. Por ello, una aproximación razonable para estimar el peso total de los cangrejos en las capturas sería multiplicar las descargas de quelas por 3 .

\section{AGRADECIMIENTOS}

Los autores agradecen las sugerencias a la redacción del manuscrito de F. Arocha, E. Villarroel y dos árbitros anónimos, así como la colaboración de los técnicos R. Marcano, G. Vizcaíno, A. Urbaneja y M. Márquez en el procesamiento de las muestras de campo y trascripción de la información a bases de datos. Así mismo, reconocemos el trabajo a bordo desempeñado por los observadores científicos C. Torrens, L. Ortiz, L. Rondón, N. Romero, E. Duarte, R. Febres, J. García, R. Rodríguez, J. Fermín, R. Antón, L. Salazar y J. Hernández, quienes recolectaron las muestras de campo. Finalmente, agradecemos la colaboración de los representantes de las empresas dedicadas a la pesca de arrastre en Cumaná y Guanta, quienes facilitaron el uso de sus embarcaciones para el desarrollo de este trabajo. El estudio fue financiado en parte por el Programa de Desarrollo Tecnológico Agropecuario, PRODETEC II, suscrito entre el Gobierno Nacional, representado por el Ministerio de Agricultura y Cría - Fondo Nacional de Investigaciones Agropecuarias (actualmente Instituto Nacional de Investigaciones Agrícolas, adscrito al Ministerio de Ciencia y Tecnología), y el Banco Interamericano de Desarrollo.

\section{RESUMEN}

Los cangrejos moros, del género Calappa, constituyen las principales especies de cangrejos desembarcados por la flota arrastrera industrial en Venezuela. Su distribución es amplia en el país, desde el Golfo de Venezuela hasta el delta del Río Orinoco, pero las mayores capturas provienen de dicho golfo, los alrededores de la Isla de Margarita y norte del estado Sucre. Las capturas en la región oriental alcanzaron $221 \mathrm{t}$ por año, con un promedio de 69 t en el período 1970-2001. Para este estudio se procesó una muestra de 2398 cangrejos moros recolectada por observadores científicos a bordo de las embarcaciones de arrastre industrial que operaron en el oriente del país entre 1994 y 2000. Tres especies componen las capturas, Calappa sulcata y C. flammea, ambas en proporción de $47 \%$, y C. nítida, que representó $5 \%$ de la muestra. La proporción de sexos estuvo fuertemente sesgada hacia los machos en C. sulcata $(2,8: 1)$ y en C. flammea $(1,9: 1)$, y ligeramente sesgada en $C$. nitida $(1,4: 1)$. Calappa sulcata es la especie con mayor interés comercial para la flota, alcanzando un peso promedio de $290 \mathrm{~g}$ y máximo de $720 \mathrm{~g}$; C. flammea alcanzó un peso promedio de 140 g y máximo de 418 g, mientras que el peso promedio de C. nitida fue $46 \mathrm{~g}$ y su peso máximo no superó los $113 \mathrm{~g}$. Los cangrejos moros son capturados de noche y de día en similar proporción en la región oriental. Aunque la distribución batimétrica de estas especies es amplia, desde pocos metros a $126 \mathrm{~m}$ de profundidad, las mayores capturas tuvieron lugar entre 38 y 54 $\mathrm{m}$. Las relaciones entre el peso de las quelas y el peso total fueron lineales y difirieron significativamente entre sexos. La relación entre el peso total y el ancho del caparazón fue alométrica, con exponente ca. 3 en C. sulcata, y menor que 3 en $C$. flamea y C. nítida. Las hembras de $C$. sulcata y $C$. nitida presentaron exponentes significativamente menores que los machos. Motivado a que las quelas son separadas a bordo para ser comercializadas, mientras que el ejemplar vivo es devuelto al mar, para fines prácticos una relación común para las tres especies, que permitiría convertir el peso de las quelas a peso total del ejemplar sería: Peso total $=3 \mathrm{x}$ peso quelas. No se observa una tendencia descendente significativa en la relación entre las capturas por unidad de esfuerzo y el esfuerzo. La variación de las capturas tiende a seguir la del esfuerzo de pesca, con una tendencia descendente de ambos parámetros en los últimos años. No se encontraron hembras ovadas en las tres especies, por lo que pudieran ocurrir migraciones reproductivas hacia aguas más profundas durante los meses de octubre a enero, cuando la abundancia de los cangrejos es menor en el área de estudio. Se recomienda que la práctica de extraer una sola quela, devolviendo el resto del animal vivo al mar, debe promoverse entre los pescadores, mientras que debería evitarse la extracción de quelas en las hembras.

Palabras claves: Calappa, pesquería de arrastre, nororiente Venezuela, biometría.

\section{REFERENCIAS}

Aravindakshan, M. y K.S. Sundaram 1983. Occurrence of the crab Calappa lophos (Herbst) in trawler landings at Sassoon Docks, Bombay. Indian J. Fish. 30: 169-170. 
Bellwood, O. 1998. The phylogeny of box crab genera (Crustacea: Brachyura: Calappidae) with notes on their fossil record, biogeography and depth distribution. J. Zool. 244: 459-471.

Chakraborty A, S.K. Otta, B.J.S. Kumar, M.S. Hossain, I. Karunasagar, V.N. Venugopal e I Karunasagar 2002. Prevalence of white spot syndrome virus in wild crustaceans along the coast of India. Curr. Sci. 82: 1392-1397.

Davis, G.E., D.S. Baugham, J.D. Chapman, D. McArthur \& A.C. Pierce 1979. Mortatlity associated with declawing stone crabs, Menippe mercenaria. Rep. SFRC T-552, Natl. Park Serv., South Fla. Res. Cent., Homestead $23 \mathrm{p}$.

Ehrhardt, N.M. \& V.R. Restrepo 1989. The Florida stone crab fishery: A reusable resource?, pp: 225-240. In: Caddy, J.F. (Ed.) Marine invertebrate fisheries: their assessment and management. Wiley, Nueva York, EEUU. 768 p.

Flegel, T. W. \& V. Alday-Sanz 1998. The crisis in Asian shrimp aquaculture: current status and future needs. J. App. Ichthyol. 14: 269-273.

Galil, B.S. 1997. Crustacea Decapoda: A revision of the Indo-Pacific species of the genus Calappa Weber, 1795 (Calappidae). Mem. Mus. Natl. Hist. Nat. France 176: 271-335.

Hameed A.S.S., G. Balasubramanian, S.S. Musthaq y K. Yoganandhan 2003. Experimental infection of twenty species of Indian marine crabs with white spot syndrome virus (WSSV). Diseases Aq. Org. 57 (1-2): 157-161.

Hayashi, T., H. Ren, T. Akiba, H. Endo \& E. Watanabe 1993. Extractive components of unutilized small crabs and their sensory evaluation as seasonings. Bull. Jap. Soc. Scien. Fish. 59: 865-873.

Hughes, R.M. \& R.W. Elner 1989. Foraging behaviour of a tropical crab: Calappa ocellata Holthuis feeding upon the mussel Brachidontes domingensis (Lamarck). J. Exp. Mar. Biol. Ecol. 133: 93-101.

Ivo, C.T.C., M.E. Santiago, M.E. \& C. Monteiro Neto 1996. By-catch in the fishery for lobsters Panulirus argus and Panulirus laevicauda, in Ceara State, Brazil. Arq. Cienc. Mar., Univ. do Ceara, Brasil. 30: 41-47.

Kuris, A.M. \& M. Mager 1975. Effect of limb regeneration on size increase at molt of the shore crabs Hemigrapsus oregonensis and Pachygrapsus crassipes. J. Exp. Zool. 193: 353-360.
Mantel, L.H. (Ed.) 1983. The Biology of Crustacea. Vol. 5. Academic Press, Nueva York, EEUU.

Muller, R. \& T. Bert 2001. 2001 Update of Florida's Stone Crab Fishery. Florida Fish and Wildlife Conservation Commission, St. Petersburg, Florida, USA.

Mendoza, J., L. Sánchez y L.A. Marcano 1994. Variaciones en la distribución y abundancia de los principales recursos explotados por la pesquería de arrastre del nororiente de Venezuela. II. Invertebrados. Mem. Fund. Cienc. Nat. La Salle 54(142): 64-81.

Nakamura, I.T. \& J. De Loyola e Silva 1992. Calappa sulcata Rathbun (Crustacea, Decapoda, Calappidae) de Guaratuba, Parana Brasil. Rev. Bras. Zool. 9: 47-51.

Restrepo, V.R. 1992. A mortality model for a population in which harvested individuals do not necessarily die: The stone crab. Fish. Bull. 90: 412-416.

Rodríguez, G. 1980. Crustáceos decápodos de Venezuela. Instituto Venezolano de Investigaciones Científicas, Caracas $454 \mathrm{p}$.

Rodríguez, G. \& H. Suárez 2002. Biodiversidad en Crustáceos. In M. Aguilera, A. Azocar \& E. González Jiménez (eds.). Ministerio de Ciencia y Tecnología, Fondo Nacional de Investigaciones Científicas y Tecnológicas. Edit. Fundación Polar, Caracas, 2 v.$1.080 \mathrm{p}$.

Rosas, C., A. Sánchez, L. Soto, E. Escobar \& A. BolongaroCrevenna 1992. Oxygen consumption and metabolic amplitude of decapod crustaceans from the northwest continental shelf of the Gulf of Mexico. Comp. Biochem. Physiol. A 101A: 491-496.

Signor, P.W. III 1985. The role of shell geometry as a deterrent to predation in terebrid gastropods. Veliger 28: $179-185$.

Takeda, M. \& T. Okutami 1983. Crustaceans and mollusks trawled off Suriname and French Guiana. Japan Marine Fisheries Resources Research Center, Japan $353 \mathrm{p}$.

Vermeij, G.J. 1982. Gastropod shell form, breakage, and repair in relation to predation by the crab Calappa. Malacologia 23: 1-12.

Villasmil, L. \& J. Mendoza 2001. La pesquería del cangrejo Callinectes sapidus (Decapoda, Brachyura) en el Lago de Maracaibo, Venezuela. Interciencia 26(7): 301-306.

Zar, J.H. 1996. Biostatiscal analysis. Prentice Hall, Nueva Jersey, EEUU. 918 p. 